\title{
LIFE TABLE TESTS OF EVOLUTIONARY THEORIES OF SENESCENCE
}

\author{
RANDOLPH M. NESSE \\ Department of Psychiatry, C 440 Med-Inn Building, University of Michigan Medical School, Ann Arbor, \\ Michigan 48109-0840
}

\begin{abstract}
The phenomenon of senescence requires both evolutionary and proximate explanations. The most widely accepted evolutionary explanation for senescence is that it never gets exposed to natural selection because environmental hazards kill all individuals before the age at which senescence causes decreased fitness. If this explanation is sufficient, wild populations should not demonstrate senescence, and their mortality rates should therefore remain constant during adult life, except when environmental causes of mortality have recently decreased. The alternative explanation for the persistence of the genes that cause senescence is that they have been selected for because they have pleiotropic effects that are beneficial early in life when the force of selection is strongest. Where this is the case, mortality rates should increase with age in wild populations. A method is described for using life table data to calculate an estimate of the intensity of selection acting on senescence in wild populations. This method is applied to a variety of life tables. The results suggest that pleiotropic genes may be important causes of senescence in some populations, but not in others. This has implications for research on the proximate mechanisms of senescence.
\end{abstract}

Key Words: senescence, aging, gerontology, evolution, evolutionary explanation, natural selection, Darwinian fitness, life tables, natural populations, intensity of selection, genes

\section{INTRODUCTION}

NATURAL SELECTION acts to decrease the frequency of genes that cause senescence and increase the frequency of genes that prevent it, but senescence obviously persists. An evolutionary explanation of why it persists is not an alternative to a proximate explanation of its physiological mechanisms, but is the necessary other half of a complete biological explanation of senescence (Mayr, 1982). The enormous efforts to understand the proximate mechanisms of senescence (Hayflick, 1985) have not been balanced by equal efforts to understand its evolution. The most widely accepted evolutionary explanation for senescence is that it is outside the reach of natural selection because predation, starvation, disease, and other environmental hazards kill essentially all wild individuals before the age at which senescence decreases fitness. This idea, first clearly formulated by Haldane (1942), was elaborated by Medawar (1957). Both em- 
phasized the importance of decreased reproduction at older ages even though environmental causes of mortality are sufficient for their thesis. Also, decreased reproduction with age is not inevitable, but must itself result from senescence. The theory that senescence persists because the genes that cause it have not been exposed to natural selection has often been thought to be sufficient (Edney and Gill, 1968; Comfort, 1979).

Alternative evolutionary explanations of senescence must, necessarily, be based on active selection for the genes that cause it. At one time it was thought that benefits of senescence to the group or species could explain its selection (Weismann, 1881), but it is now recognized that natural selection is weak at these levels of organization and cannot sustain traits that are significantly detrimental to individuals, even if they benefit the species (Williams, 1966). Another reason that senescence-causing genes could be selected for, first proposed by Williams (1957), is that they may have pleiotropic effects that offer benefits early in adult life when the force of selection is the strongest. The force of selection declines steadily with increasing age because the number of surviving (and reproducing) individuals is lower in every successive age group as the result of death of some proportion each year from environmental hazards. Thus, a pleiotropic gene that offers a small benefit to many individuals in youth may be selected for even if it severely decreases fitness in later life, where it will have an effect on only a few individuals (Williams, 1957; Nesse, 1987). As an example, Williams suggested a hypothetical gene that alters calcium metabolism in a way that both strengthens bones in youth and occludes arteries in old age. In his original paper, he cites several lines of evidence in support of a pleiotropic explanation of senescence. He predicted that the age of reproductive maturity should mark the onset of senescence, a finding that has been amply confirmed, and that is supported by human data that show the peak of athletic ability and the valley of mortality rates in the late teens and early twenties (Fries and Crapo, 1981). Williams noted that sex differences in senescence rates can be explained by sex differences in mortality factors and fecundity, and that "there should be little or no post-reproductive period in the normal life-cycle on any species" (Williams, 1957, p. 407). In animals that care for their young, the post-reproductive period begins only after the young no longer benefit from parental efforts. In animals that do not care for their young and that have high mortality rates between widely separated breeding seasons, pleiotropic effects will be especially beneficial and can be expected to have major influence on senescence. In extreme cases, such as salmon, high interbreeding period mortality rates make natural selection very weak after breeding. This results in a semelparous life-cycle in which breeding takes place once at a peak of vitality, and then the deleterious effects of pleiotropic genes are expressed all at once (because natural selection cannot oppose them) and death from senescence occurs within days. Although Williams emphasized selection for genes that cause senescence but provide a small benefit in youth and a large cost in old age, senescence could also result from selection against anti-aging genes (that repair or delay age-associated tissue damage) because they impose a small cost in youth (Nesse, 1987). Thus, selection against senescence and for longevity (Cutler, 1979) is opposed by selection for the pleiotropic benefits of genes that cause senescence, and selection against the pleiotropic costs of genes that promote longevity.

Breeding experiments and mathematical models have confirmed the existence of pleiotropic genes that influence senescence. Selection for late reproduction in female fruit flies increases longevity, decreases early fecundity, and increases late fecundity 
(Rose and Charlesworth, 1980; Rose, 1984; Luckenbill et al., 1984). Selection for early reproduction decreases longevity in flour beetles (Sokal, 1970). Hamilton provided a mathematical model for the natural selection of age-specific fitness effects and concluded that "for organisms that reproduce repeatedly, senescence is to be expected as an inevitable consequence of the workings of natural selection" (Hamilton, 1966, p. 26). Charlesworth (1980) has incorporated senescence into a general treatment of agespecific fitness effects, and Rose (1985) has developed a model of life histories with overlapping generations in which substantial variation in rates of senescence is maintained by pleiotropic effects. Despite these reports, the pleiotropic theory of senescence has not been widely recognized. This may be because field evidence seems to support the nonexposure to selection theory, because it is not understood that an evolutionary explanation of senescence is necessary in addition to a proximate explanation of its mechanisms, or because the pleiotropic theory implies that the mechanisms of aging are likely to be diverse and intrinsic to fundamental bodily processes in ways that preclude substantial modification.

Although the pleiotropic and the nonexposure to selection theories of senescence are not mutually exclusive, they do represent two distinct evolutionary explanations for senescence. Determination of their relative contributions is important because we may soon be able to modify the effects of genes that cause senescence. If some of these genes are of the pleiotropic type described by Williams, such efforts may interfere with beneficial effects in youth. Also, a careful search for possible beneficial effects associated with diseases of aging, such as Alzheimer's Disease, will be justified only if pleiotropic genes are shown to make important contributions to senescence. Finally, the evolutionary explanation of senescence may have broad implications for our understanding of our own motives and mortality (Alexander, 1987; Nesse, 1987).

The two theories make different predictions about the effects of senescence on fitness in wild populations in ecologically stable habitats. If senescence is outside the reach of natural selection because it does not decrease Darwinian fitness during the usual life span in the wild, then mortality rates should remain constant during all of adult life. If pleiotropic effects are important, however, then mortality rates should increase with age in wild populations. Thus, where senescence does not affect wild populations, pleiotropic effects are not important, but where senescence does decrease fitness in the wild, this cannot be explained by the nonexposure to selection theory, and pleiotropic effects are likely to be important. These predictions make it possible to assess the relative contributions of the two factors from quantitative estimates of the effect of senescence on Darwinian fitness in wild populations.

\section{MATERIALS AND METHODS}

A method was developed to calculate, from life table data, the intensity of selection (I) acting against senescence. Life table data for an actual wild population are used to construct another life table for a hypothetical population that is identical except that senescence does not occur, that is, the adult mortality rate is assumed to be constant at the rate observed in the actual population at the age of sexual maturity. Darwinian fitness is proportional to the total number of individual reproductive years $(I R Y)$ in each population. The relative reproductive advantage for the hypothetical population without senescence is given by the ratio of the $I R Y$ in the hypothetical population $\left(I R Y_{h}\right)$ to 


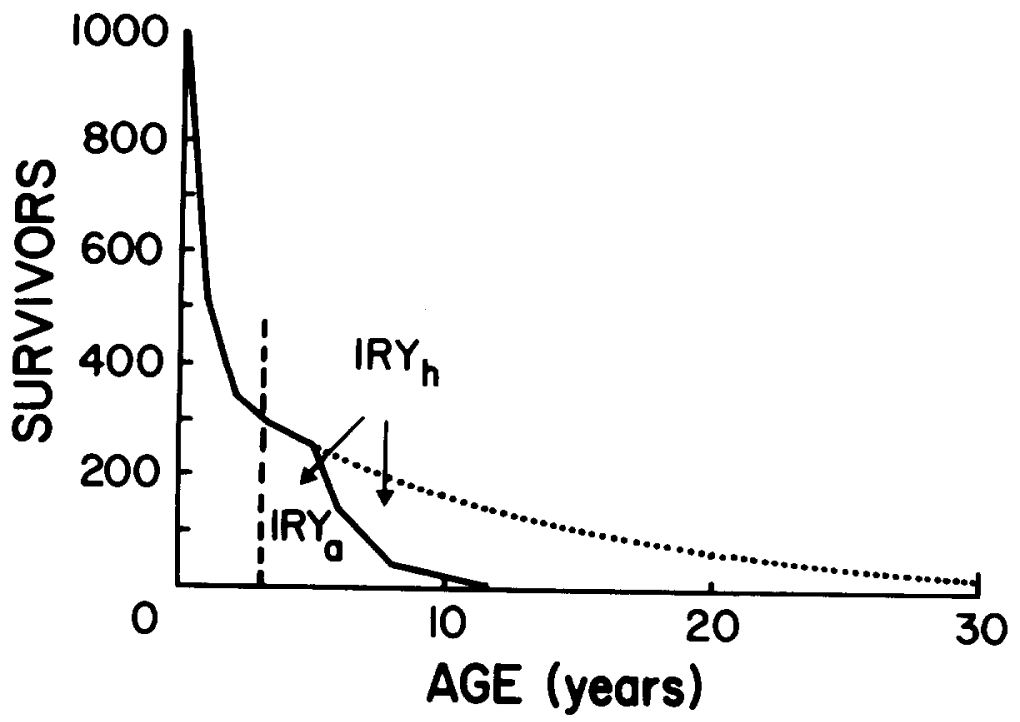

FIG. 1. Survival curves of male impala (solid line) and a hypothetical population without senescence (dotted line). Vertical dashed line is the age at full sexual maturity, vertical solid line is the age at which only $2.5 \%$ of the population remains.

the $I R Y$ in the actual population $\left(I R Y_{a}\right)$. The intensity of selection is the percent decrease in fitness imposed by senescence compared to the hypothetical group and can be calculated by $I=\left(I R Y_{h}-I R Y_{a}\right) \div I R Y_{h}$ (Van Valen, 1965). Because many genes are involved in senescence, it is not feasible to calculate absolute rates of change in gene frequency, but $I$ is proportional to the force of selection acting on senescence and therefore to the number of generations required for a specified change in gene frequency (Bodmer and Cavalli-Sforza, 1976). All analyses are based on life tables adjusted to an initial population of 1000 . Standard definitions of survivors $(l x)$, mortality rate $(q x)$, and age structure $(L x)$ are used (Deevey, 1947). The method assumes, for simplicity, that the reproduction rate after maturity is constant. This favors the nonexposure to selection theory if reproductive capacity decreases with age as a result of senescence. In order to ensure inclusion of the risks of mate competition and reproduction, the baseline adult mortality rate in the group without senescence is assumed to be equal to the rate observed in the actual population at the age of full sexual maturity. In order to avoid undue influence of any one data point, the $q x$ at sexual maturity for the hypothetical group is derived from actual $q x$ values smoothed by Hanning (Tukey, 1977). Summation of $I R Y$ values is based on unsmoothed values of $L x$. Values representing less than $2.5 \%$ of the original population are excluded because of their possible unreliability. This convention also favors the nonexposure to selection theory. A variety of published life tables for wild populations were analyzed and graphed using a microcomputer-integrated spreadsheet/graphics program. Despite an extensive literature search, too few high-quality life tables were found to allow random sampling, so selection of tables was based only on their relative freedom from sampling bias, age determination errors, marker loss, and artificial or temporary factors that change mortality rates. 
TABle 1. THE INTENSITY OF SELECTION ACTING ON SENESCENCE IN WILD POPULATIONS

\begin{tabular}{|c|c|}
\hline Species & $I$ \\
\hline Dall sheep (Ovis d. dalli) (Deevey, 1947) & .86 \\
\hline Waterbuck (Kobus Defassa ugandae Neumann) male (Spinage, 1970) & .64 \\
\hline Impala (Aepyceros melampus Lichenstein) male (Spinage, 1972) & .69 \\
\hline female & .62 \\
\hline Buffalo (Syncerus caffex Sparrman) male (Spinage, 1972) & .79 \\
\hline female & .60 \\
\hline Caribou (Rangifer arcticus arcticus Richardson) (Banfield, 1955) & .05 \\
\hline Himalayan Thar (Hemitragus Jemlahicus) female (Caughley, 1966) & .18 \\
\hline Zebra (Equs burchelli boehmi) male (Spinage, 1972) & .41 \\
\hline female & .60 \\
\hline Black rhinoceros (Diceros bicornis) (Goddard, 1970) & .24 \\
\hline Hippopotamus (Hippopotamus amphibius) (Laws, 1968) & .75 \\
\hline African elephant (Loxodonta africana) female (Corfield, 1973) & .40 \\
\hline Herring gull (Larus argentatus smithsonianus) (Paynter, 1966) & .04 \\
\hline Lapwing (Venellus vanellus) (Lack, 1954) & .06 \\
\hline Great tit (Parus major) male (Bulmer, 1973) & .26 \\
\hline female & .06 \\
\hline North American Mallard (Anas platyrhynchos) (Hickey, 1972) & -.03 \\
\hline Blue jay (Cyanocitta cristata) (Bulmer and Perrins, 1973) & .02 \\
\hline Lake trout (Cristivomer namaycush) (Deevey, 1947) & .22 \\
\hline Sessile rotifer (Floscularia coniferia) (Edmondson, 1945) & .60 \\
\hline Barnacles (Balanus balanoides) (Deevey, 1947) & .57 \\
\hline Human, USA, 1970 (Homo Sapiens) (Vital Statistics of U.S.A., 1974) & .85 \\
\hline
\end{tabular}

\section{RESULTS}

Figure 1 illustrates this method of analysis using data on male impalas. For the actual population, the mortality rate decreases from birth up to the fourth year and then increases sharply. The actual survival curve declines more rapidly as the mortality rate increases with age, while the survival curve for the hypothetical population declines steadily and more slowly. The area enclosed by the actual survival curve, the vertical line that indicates the age of first reproduction, and the vertical line at the age when only $2.5 \%$ of the population remains (equal to $I R Y_{a}$ ), is far smaller than the corresponding area enclosed by the hypothetical survival curve (equal to $I R Y_{h}$ ). This reflects the threefold reproductive advantage of a hypothetical individual without senescence over the average actual individual. The coefficient of selection acting against senescence for this wild population is $\mathbf{0 . 6 8 3}$.

Table 1 summarizes the results for a variety of species. For some, especially birds, senescence appears to have little effect on fitness and therefore gives no indication that pleiotropic effects contribute to senescence. For many species, however, the coefficient of selection is remarkably high, and suggests strong selection against senescence. For these populations, the nonexposure to selection theory is insufficient and pleiotropic effects are likely to be important causes of senescence.

\section{DISCUSSION}

Uncertainty about these conclusions arises mainly from uncertainty about the stability of mortality rates and the effects of rapid environmental changes. If an ecological 
change, such as elimination of a major predator, drastically decreases mortality rates, then senescence observed in a wild population may result, not from pleiotropic genes, but from genes that are newly exposed to selection. There are several reasons to think that recently decreased mortality rates cannot account for all of the senescence observed in wild populations. First, the existence of pleiotropic genes that cause senescence is predicted by populations genetics (Hamilton, 1966) and has already been confirmed by breeding experiments (Rose, 1984). Second, it seems unlikely that barnacles and rotifers, as well as the many larger species described, have all recently experienced an extraordinary decrease in mortality rates. For nine of the species reported here, the mortality rate from environmental hazards would need to more than double during the entire lifespan in order to reduce the intensity of selection acting on senescence below .02. For many of the populations studied, senescence reduced reproductive success more than all environmental hazards combined. Finally, the possibility that senescence observed in wild populations is an artifact of artificially decreased mortality rates seems less likely than the converse. When human or other environmental factors increase mortality rates, then the data will appear to support the nonexposure to selection theory, even if pleiotropic effects are present. The data currently available are inadequate to allow a firm conclusion about the importance of these possible sources of error. The problem of the evolution of senescence provides an important reason for collecting detailed life tables on as many wild species as possible.

Why the extent of pleiotropic contributions to senescence should vary so greatly in different species is a matter for further study. The available data suggest that pleiotropic effects are minimal in birds and are important in large mammals, but severe limitations of the available data make even these generalizations preliminary. It is possible that the intensity of selection acting on senescence for a given species could be a useful life history trait that offers clues about its recent evolutionary history. For instance, a species that has recently been released from predation pressure or has found new sources of food or shelter would experience an increased life span, and the age of expression of any existing pleiotropic and other senescence causing genes would be pushed, by natural selection, later and later in life in a tighter and tighter cluster. In the laboratory, such a species should show a relatively large number of genes contributing to senescence and a relatively steep increase in mortality rates with a resulting narrow range of life spans. Conversely, a species newly subject to environmental changes that decrease life span would be expected to gradually accumulate pleiotropic and other genes that cause senescence. Such species should have only a few genes involved in senescence and should show a relatively variable life span, even in the laboratory.

The distinction between the two theories of the evolution of senescence is also important for proximate studies. Aspects of senescence that derive from pleiotropic genes would be expected to make relatively large contributions to senescence and may require study in ways distinct from those that persist simply because they have not been exposed to selection. In particular, aspects of senescence that actively damage tissue are especially likely to be maintained by pleiotropic effects, while the absence of mechanisms to protect against and repair environment induced damage, is more likely to be explained by nonexposure to selection. Autoimmune tissue damage, for instance, occurs with some frequency in early adulthood where it is subject to selection, and it is therefore likely to be maintained by pleiotropic benefits such as the ability to aggressively resist infection. On the other hand, genes that would allow growth of a third set of 
teeth in humans have probably simply not been subject to selection, although it is conceivable that early detrimental pleiotropic effects, even if small, have resulted in selection against such genes. Genes that cause cancer may be selected for because they enhance the ability to regenerate damaged tissue. At present only tentative conclusions are possible about even the relative importance of the two evolutionary explanations of senescence for any given species, and research has not yet begun to try to determine which aging mechanisms result from pleiotropic genes and which have simply never been exposed to selection.

The greatest current contribution of this approach may be simply to note that these two evolutionary explanations are not mutually exclusive and together are almost certainly sufficient to explain the evolution of all the mechanisms that cause senescence. Are other evolutionary explanations possible? The genes responsible for senescence must either be outside the reach of selection or must be actively selected for because they benefit an individual's inclusive fitness. If they benefit individual fitness, it could only be as a result of pleiotropic effects, since their effects late in life are, by definition, detrimental. The main alternative possibility is that natural selection is limited in its ability to eliminate senescence because the necessary mutations have not occurred, or because of the random factors in the process of selection. If this were the case, one would expect to find differences in the rate of decline of different organ systems, but the reserve capacities in organ systems decline with age along exactly parallel and exponential trajectories starting at reproductive maturity (Strehler and Mildvan, 1960). This is exactly what one would expect if the various mechanisms that control aging have been subject to a force of selection that declines exponentially from maturity on. The "One Hoss Shay" falls apart all at once because the force of natural selection declines rapidly at the age at which some effects of senescence severely decrease fitness, so other effects of senescence accumulate at the same age and the force of selection declines even more abruptly.

Robust correlations have been demonstrated between the life spans of different species and their ability to repair DNA, their level of protection against damaging superoxides, and the number of cell divisions possible (Cutler, 1982). Although such correlations demonstrate that these mechanisms are important factors in the aging that is observed in protected settings, they may even more dramatically demonstrate that natural selection has successfully shaped protective mechanisms that prevent these aspects of senescence from decreasing fitness during the normal lifespan in the wild, and the reason that these defenses are not more effective is because selection has not had a chance to shape their effects at older ages.

The declining force of natural selection with increasing age is at the core of both evolutionary explanations of senescence. This decline is also responsible for the apparent coordination of different aspects of aging within a species, and for the correlations between life spans and aspects of aging between species. It is not necessary to postulate a central mechanism that controls the expression of the effects of aging - the declining force of selection is a sufficient explanation. In combination with the growing recognition that no single effect of aging is primary (Hayflick, 1985), it appears somewhat unlikely, theoretically as well as practically, that it will be possible to extend the maximum life span. It remains likely, however, that increased research on aging will lead to insights that advance proximate gerontologic research in ways that will advance our ability to treat the diseases of aging. 
Acknowledgments - I thank Armen Asherian and Alan Rosenberg for collecting and analyzing many of the life tables, and members of the University of Michigan Evolution and Human Behavior Program, and the Lutz Street Group for their helpful comments. This work was supported by the Evolution and Human Behavior Program and the Department of Psychiatry at the University of Michigan.

\section{REFERENCES}

ALEXANDER, R.D. The Biology of Moral Systems. Aldine Press, New York, 1987.

BANFIELD, A.W.F. A provisional life table for the barren ground caribou. Can. J. Zool. 33, 143-147, 1955.

BODMER, W.F. and CAVALLI-SFORZA, L.L. Genetics, Evolution and Man. W.H. Freeman, San Francisco, 1976.

BULMER, M.G. and PERRINS, C.M. Mortality in the great tit parus major. Ibis. 115(2), 277-281, 1973.

CAUGHLEY, G. Mortality patterns in mammals. Ecology. 47(6), 906-918, 1966.

CHARLESWORTH, D. Evolution in Age Structured Populations. Cambridge University Press, Cambridge, England, 1980.

COMFORT, A. The Biology of Senescence, 3rd Ed. Elsevier Press, New York, 1979.

CORFIELD, T.F. Elephant mortality in Tsavo National Park, Kenya. East Afr. Wildl. J. 11(3-4), 339-368, 1973.

CUTLER, R.G. Evolution of human longevity: A critical overview. Mech. Ageing Dev. 9, 337-354, 1979.

CUTLER, R.G. Longevity is determined by specific genes: Testing the hypothesis. In: Testing the Theories of Aging, Adelman, R.C. and Roth, G.S. (Editors), pp. 24-114. CRC Press, Inc., Boca Raton, FL 1982.

DEEVEY, E.S. Life tables for natural populations of animals. Q. Rev. Biol. 22, 283-314, 1947.

EDMONDSON, W.T. Ecological studies of sessile rotatoria Part II - Dynamics of populations and social structures. Ecol. Monogr. 15(2), 141-172, 1945.

EDNEY, E.B. and GILL, R.W. Evolution of senescence and specific longevity. Nature. 220, 281-282, 1968.

FRIES, J.F., and CRAPO, L.M. Vitality and Aging. W.H. Freeman and Co., San Francisco, 1981.

GODDARD, J.E. Age criteria and vital statistics of a black rhinoceros population. East Afr. Wildl. J. 8, $105-121,1970$.

HALDANE, J.B.S. New Paths in Genetics. Harper, London, 1942.

HAMILTON, W.D. The moulding of senescence by natural selection. J. Theor. Biol. 12, 12-45, 1966.

HAYFLICK, L. Theories of biological aging. Exp. Gerontol. 20, 145-159, 1985.

HICKEY, J.J. Survival studies of banded birds. U.S. Fish and Wildife Service Special Scientific Report Wildlife No. 15, 1952.

LACK, D. The Natural Regulation of Animal Numbers. Oxford Press, London, 1954.

LAWS, R.M. Dentition and aging of the hippopotamus. East Afr. Wildl. J. 6, 19-51, 1968.

LUCKENBILL, L.S., ARKING, R., CLARE, M.J., CIROCCO, W.C., and BUCK, S.A. Selection for delayed senescence in Drosophila Melanogaster. Evolution, 38, 996-1003, 1984.

MAYR, E. The Growth of Biological Thought. Belknap Press, Cambridge, MA, 1982.

MEDAWAR, P.B. Old age and natural death. In: Medawar, P.B. (Editor), The Uniqueness of the Individual. Methuen and Co., London, 1957.

NESSE, R.M. An evolutionary perspective on senescence. In: Ethical Dimensions of Geriatric Care, Spicker, S.F., Ingram, S.R. and Lawson, I.R. (Editors), pp. 45-64. D. Ridel Publishers, Boston, 1987.

PAYNTER, R.A. Jr. A new atempt to construct lifetables for Kent Island herring gulls. Bull Mus. Comp. Zool. 133(11), 489-528, 1966.

ROSE, M.R. and CHARLESWORTH, B. A test of evolutionary theories of senescence. Nature 287, 141$142,1980$.

ROSE, M.R. Laboratory evolution of postponed senescence in Drosophila Melanogaster. Evolution 38, 1004-1010, 1984.

ROSE, M.R. Life history evolution with antagonistic pleiotropy and overlapping generations. Theor. Pop. Biol. 28, 342-358, 1985.

SPINAGE, C.A. Population dynamics of the Defassa waterbuck (Kobus Defassa Ugandae Neumann) in the Queen Elizabeth Park. J. Anim. Ecol. 39(1), 51-78, 1970.

SPINAGE, C.A. African ungulate life tables. Ecology 53(4), 645-652, 1972.

SOKAL, R.R. Senescence and the genetic load: Evidence from Tribolium. Science 167, 1733-1734, 1970. STREHLER, B.L. and MILDVAN, A.S. General theory of mortality and aging. Science 132, 14-21, 1960. 
TUKEY, J.W. Exploratory Data Analysis. Addison-Wesley, Reading, MA, 1977.

VAN VALEN, L. Selection in natural populations - III, Measurement and estimation. Evolution 19, 514 528, 1965.

Vital Statistics of the United States, 1970. National Center for Health Statistics, Rockville, MD, 1974.

WEISMANN, A. The duration of life. In: Poulton, E.B., Seho, and Shipley, A.E. (Editors), A Weismann:

Essays upon Heredity and Kindred Biological Problems. Clavedon Press, Oxford, England, 1889.

WILLIAMS, G.C. Pleiotropy, natural selection and the evolution of senescence. Evolution 11, 398-411, 1957.

WILLIAMS, G.C. Adaptation and Natural Selection. Princeton University Press, Princeton, NJ, 1966. 